\title{
COOPERACIÓN ENTRE INSTITUCIONES DE EDUCACIÓN SUPERIOR Y DE ENSEÑANZA MEDIA TÉCNICO-PROFESIONAL: LA FORMACIÓN ÉTICA COMO COMPETENCIA LABORAL
}

\section{Modelo educativo DuocUC: competencias de empleabilidad y programas transversales}

El Instituto Profesional DuocUC definió su misión institucional como "la formación de personas en el área técnico-profesional con una sólida base ética, capaces de actuar con éxito en el mundo laboral y comprometidas con el desarrollo del país". Esta declaración implica reconocer una finalidad propia que se expresa, en términos de misión, propósitos y proyecto educativo, como una "educación para el trabajo", basada en una "enseñanza práctica", con énfasis de conocimientos aplicados y el desarrollo de habilidades y destrezas directamente relacionadas con el ejercicio de un oficio determinado. Pero, además, con un sello distintivo: la formación ética de los alumnos como expresión del ejercicio de virtudes profesionales.

Lo anterior implicó adoptar una metodología propia a su singularidad funcional, es decir, medios —organización, gestión, procesos educativos, de diseño curricular, de formación y de evaluación- especialmente adecuados a tales fines.

En este sentido, su calidad de "formación" se encuentra directamente relacionada con su capacidad de aportar valor laboral, social y cultural a sus egresados. Su unidad de medida debe corresponder a la forma en que esa productividad sea reconocida. La expresión "competencias laborales" constituye el estándar que 
se ha ido imponiendo en los diversos modelos de medición de productividad enfocados al desempeño concreto de un individuo en un contexto laboral determinado, especialmente utilizados en procesos de mejoramiento continuo y certificación de calidad.

De este modo, el énfasis práctico de la enseñanza que DuocUC proporciona significa concebir el objetivo central de todo el proceso formativo - expresado en el perfil de egreso de cada especialidad - como un conjunto de "competencias", cuya identificación corresponda a un proceso de prospección de las referidas exigencias de la industria.

Con todo, un modelo de esta singularidad, y de acuerdo con los estándares ya habituales en el mundo, reconoce la coexistencia de dos grandes tipos o grupos de competencias: las genéricas, básicas o de empleabilidad, aquellas que favorecen la inserción laboral, la continuidad en el empleo y hasta la posibilidad de movilizarse al interior del sistema, y las competencias laborales propiamente tales, aquellas que expresan las exigencias específicas de desempeño por tipo de trabajo o actividad.

Para asegurar la implementación homogénea y efectiva de programas conducentes a la transferencia de competencias genéricas o de empleabilidad, se constituyeron unidades centralizadas responsables de definir los propósitos y alcances de cada uno de ellos, así como la adopción de una estrategia metodológica apropiada. En efecto, el Plan Estratégico Institucional 20002005 contempló la creación de programas transversales, a cargo del desarrollo e implementación de los modos instruccionales más efectivos y eficaces para transferir estas competencias universalmente reconocidas: Inglés, Matemáticas, Institucional de Cursos en Línea e Interacciones TIC, Emprendimiento y Formación General (PFG). 


\section{El Programa de Formación General (PFG)}

El PFG es la expresión académica de la misión DuocUC de "formar personas en el área técnico-profesional con una sólida base ética". Representa una forma sistemática de llevar a cabo la dimensión formativa del modelo educativo propuesto en dicha misión.

Responde también a las exigencias de la industria actual, que ve en las habilidades de comunicación y comportamiento en general, en las relaciones interpersonales y en la ética, como ejercicio de virtudes profesionales, competencias laborales estándar.

En su fundamentación siguió el modelo antropológico cristiano, según su expresión occidental clásica, y sus ejes de implementación se basaron en el programa Ethics Across the Curriculum, del Utah Valley Community College (USA). Cualificando las conductas de entrada, el PFG transmite conocimientos básicos y desarrolla habilidades generales de comportamiento (competencias laborales blandas). Además, otorga importancia central a la voluntad y se orienta a la toma de decisiones y a la capacidad de asumir relaciones laborales de una determinada manera.

El PFG mide sus resultados por la diferencia entre las conductas de entrada individuales y el nivel de logro de los objetivos terminales de cada curso (competencias). Es una secuencia curricular progresiva, de cuatro etapas:

- Desarrollo de la expresión oral y escrita según criterios argumentales lógicos (uso de la lengua nativa, desarrollo del pensamiento crítico y comunicación eficaz).

- Desarrollo del autoconocimiento, habilidades de relación y autodisciplina.

- Desarrollo de la ética personal, la integridad y la honestidad.

- Desarrollo de conciencia profesional e identificación con la compañía. 


\section{Diversidad educacional en DuocUC: el Liceo Politécnico Andes}

DuocUC reconoce diversas necesidades y oportunidades educacionales. Así, de acuerdo a la Ley Orgánica Constitucional de Enseñanza (LOCE) y aparte de otras modalidades referidas a capacitación y educación continua, organiza su proyecto educativo a través de un instituto profesional (IP), un centro de formación técnica (CFT) y un liceo de enseñanza media técnico-profesional (EMTP).

En efecto, el Liceo Politécnico Particular Andes es un establecimiento educacional de EMTP, de la Fundación Duoc de la Pontificia Universidad Católica de Chile, que funciona desde 1990 en la comuna de Renca en Santiago de Chile.

Actualmente, el liceo tiene la calidad de "plantel de especial singularidad", lo que le permite impartir, desde $1^{\text {er }}$ año de enseñanza media, las especialidades de Electrónica (orientada fundamentalmente a Control Automático), Telecomunicaciones (referida a Conectividad y Redes) y Mecánica Automotriz (con fuerte énfasis en Electrónica Automotriz y Autotrónica).

En 2005 la matrícula del liceo alcanzó los 1.270 alumnos, distribuidos por especialidad de la siguiente manera: 333 en Electrónica, 531 en Telecomunicaciones y 406 en Mecánica Automotriz.

Desde 1993 (primera promoción de egreso) hasta 2004 han egresado 2.815 alumnos. De éstos, han obtenido el título profesional de técnico de nivel medio 2.551 alumnos, lo que representa el 90,66\% del total. El siguiente cuadro muestra la distribución de estos resultados: 


\begin{tabular}{|l|c|c|c|}
\hline \multicolumn{1}{|c|}{ ESPECIALIDAD } & EGRESADOS & TITULADOS & $\%$ TITULADOS \\
\hline Secretariado & 529 & 506 & $95,65 \%$ \\
\hline Procesos Industriales & 1.017 & 906 & $75,88 \%$ \\
\hline Mecánica Automotriz & 710 & 638 & $89,90 \%$ \\
\hline Electrónica & 220 & 178 & $80,91 \%$ \\
\hline Telecomunicaciones & 339 & 323 & $95,28 \%$ \\
\hline TOTAL & 2.815 & 2.551 & $90,62 \%$ \\
\hline
\end{tabular}

Tratándose de un liceo técnico-profesional, la inversión en plataforma tecnológica para cada una de las especialidades ha sido una prioridad institucional, habiendo alcanzado altos estándares en tal sentido. Sin embargo, un elemento fundamental de su proyecto educativo apunta a desarrollar acciones que permitan potenciar la formación personal de los alumnos. De ese modo, no se sustrae a la misión general de la institución y trabaja, en su nivel y de acuerdo a su singularidad específica, en imprimir en sus alumnos el mismo sello distintivo que DuocUC promueve en el IP y en el CFT: la formación para el ejercicio de virtudes en el trabajo, con base en una antropología que ve al hombre como centro y raíz de todas las actividades humanas.

Desde 2003 se ha implementado una serie de iniciativas conjuntas y de cooperación entre la Dirección de Formación General, a través del PFG, y el Liceo Politécnico Particular Andes, tendientes a materializar la transferencia de este subgrupo de competencias genéricas que dicen relación con el sello distintivo del proyecto general de la institución.

\section{Bases estratégicas de la cooperación}

Las siguientes iniciativas o proyectos se han puesto en práctica desde el año 2003:

a) Talleres de formación en virtudes para alumnos ( $1^{\circ}$ a $4^{\circ}$ medio).

b) Conferencias sobre temas de relevancia ético-social para alumnos y docentes.

c) Escuela para padres.

d) Talleres para docentes. 
La adecuación de los objetivos instruccionales del PFG al liceo dice relación con la naturaleza de este último y con el estadio vital o etapa formativa en que se hallan sus alumnos. Sin embargo, responde también a una estrategia educacional de largo plazo, favorecida por la articulación, en DuocUC, de las tres categorías en las que implementa su proyecto.

El liceo, si bien es de sostenedor laico, al pertenecer a la Fundación DuocUC de la Pontificia Universidad Católica de Chile se enmarca, naturalmente, en la misión evangelizadora de ésta. Con todo, y dada la naturaleza del PFG y la consideración de la ética como competencia laboral estándar exigida hoy en diversos sectores productivos, el trabajo en pro de la transferencia de las competencias genéricas opera en un contexto de ética natural y no confesional.

Por otra parte, la condición socioeconómica y cultural de los alumnos del liceo corresponde a un promedio bajo, lo que incide fundamentalmente en la educación reflejada de cada grupo familiar. Ello implica la alta frecuencia de hogares disgregados, disfuncionales y disvalóricos, lo que hace particularmente compleja la transferencia desde lo que podríamos denominar un "perfil de ingreso". Por esta razón, el quehacer formativo del liceo toma un acento particular, que lo diferencia de lo desarrollado en el IP y CFT DuocUC, en tanto asume un carácter "remedial" y, hasta cierto grado, subsidiario.

No obstante, abordar la formación de valores y el desarrollo de virtudes a través de estrategias formativas consistentes con un modelo educativo basado en competencias laborales no es cosa fácil. Los profesores del liceo — principales ejecutores de la iniciativa - tienen, en general, claridad conceptual o académica sobre estos temas, pero carencias en el enfoque metodológico para transferir estas competencias en los alumnos. Para ello ha sido necesario desarrollar un lenguaje profesional específico que permita potenciar la transferencia de los valores y las virtudes y 
su efectiva aplicación a la gestión, considerados como factores de productividad.

La verdad es que este enfoque tiene de nuevo sólo el nombre, pues es una conceptualización muy certera de una ética clásica de ejercicio de las virtudes frente a las llamadas "éticas de la convicción" y, desde luego, de las éticas de resultados meramente utilitaristas. En efecto, la ética no es un tema académico o una abstracción filosófica, sino un conjunto de acciones orientadas a un fin, que es la perfección de la naturaleza humana. Es decir, la ética, al tener que ver con las acciones humanas, afecta a aquellas que atañen al mundo del trabajo y, por ende, al lugar donde hoy éste se desarrolla preferentemente: la empresa.

Lo que ocurre es que el desarrollo conceptual de las organizaciones económicas tomó cierto tiempo en reasumir su rasgo de corporaciones humanas, sumidas aún en criterios y enfoques residuales de la revolución industrial. Cuando nos referimos al trabajo, tanto la ética como la productividad son sólo distintas dimensiones de una misma realidad en la organización. La ética no tiene por objeto principal la formulación de normas o de prohibiciones, sino que su fin es generar en las personas la capacidad de realizar la buena acción que, en cuanto tal, debe conseguir los resultados esperados (debe ser eficaz) y debe ser coherente con los recursos de los que dispone la organización (debe ser eficiente). Por lo tanto, la incorporación de la ética en la empresa (y en un proyecto educativo que procura que sus planes de estudio sean pertinentes a los requerimientos de aquélla) es la base de una cultura organizacional que se distinga por su eficiencia y su eficacia. La ética es fundamento de virtudes empresariales y no simplemente de la formulación de códigos de conducta. Por ello, resulta adecuado hablar de la "rentabilidad de la ética", no respecto a su fundamentación, claro está, sino en cuanto su externalidad principal, mensurable no sólo cualitativa sino también cuantitativamente. 
Entonces, aparte del papel remedial y/o subsidiario que, por carencias de origen, el liceo ha debido abordar en materias formativas, y del contexto debido a su naturaleza como establecimiento EMTP, el trabajo desarrollado por el PFG, en conjunto con el plantel, ha tenido por objetivo principal hacer ver a los alumnos la necesidad que tiene la empresa actual de que sus miembros sean principalmente profesionales éticos; pero no en el sentido de que asuman determinados principios valóricos, sino que los pongan efectivamente en práctica. $\mathrm{Y}$, además, motivarlos para el desarrollo y la ejecución futura de la acción éticamente buena, mostrándoles en qué sentido, siendo ellos más éticos, son mejores profesionales, mejores personas y, consecuentemente, más felices.

\section{El proyecto en particular}

\subsection{Taller de desarrollo personal en virtudes}

Este proyecto se ha desarrollado para complementar la formación de los alumnos del liceo, especialmente en lo relativo al uso de la libertad, mediando sus facultades superiores como personas (inteligencia y voluntad). Por lo mismo, se hace necesario contextualizar su implementación, de modo de ilustrar el tipo de perspectiva tenido a la vista — con cierto carácter estratégico-.

Como se señalara anteriormente, y teniendo presente que la formación del juicio moral de los jóvenes se adquiere en forma natural a través de la convivencia familiar -o en cierto modo "fenomenológico" a través del intercambio experiencial con los pares-, resulta clave considerar la interacción con distintos agentes de la sociedad. Cuando esto no se logra, el liceo asume una función de carácter subsidiario. Sin embargo, es necesario considerar que hay elementos o factores que conspiran contra la familia en la educación de los hijos, que se han ido potenciando en los últimos años. Podemos destacar los siguientes: 
- La generalizada importancia atribuida al éxito y al lucro, que conspira contra los padres guiándolos equivocadamente en el planteamiento de sus prioridades de vida familiar, resta involucramiento en la educación de los hijos y mengua el tiempo dedicado a ello, todo lo cual debilita el vínculo que confiere fuerza a la tarea educativa.

- La madre comparte menos tiempo en la casa con los hijos, debilitando la supervisión y guía.

- La televisión se ha constituido en un ente creador de modelos de conducta para la juventud, mostrando no pocas veces una visión falseada de la vida y hasta difundiendo valores reñidos con lo humano.

- Aumenta progresivamente la tasa de rupturas matrimoniales y la de hijos criados por un solo progenitor (como sabemos, cerca del $48 \%$ de los niños nacidos en Chile lo hacen fuera del matrimonio y de la familia naturalmente constituida).

- Síntomas de deterioro conductual progresivo en la sociedad, dificultando la labor educadora de los padres. En particular, el aumento de la violencia y la drogadicción juvenil, el creciente desprecio a toda manifestación de autoridad, el deterioro del lenguaje y un reconocimiento cada vez menor a las bases antropológicas de la sexualidad.

Considerando lo anterior, los talleres de desarrollo personal en virtudes se implementaron con los siguientes objetivos:

a) Transferir al alumno herramientas concretas básicas que les permitan ejercer las virtudes, en el marco de los objetivos transversales de la institución.

b) Ayudar al alumno a tomar conciencia de que su autodesarrollo y autosuperación son aspectos importantes para ser mejor persona.

c) Transferir a los alumnos de $1^{\circ}$ y $2^{\circ}$ medio, dada la etapa de su desarrollo personal, la capacidad de desplegar tres buenos hábitos operativos: fortaleza, templanza y honestidad. 
d) Transferir a los alumnos de $3^{\circ}$ y $4^{\circ}$ medio, dada la etapa de su desarrollo personal, la capacidad de desplegar tres buenos hábitos operativos: respeto, prudencia y reconocimiento de la dignidad de la persona.

e) Incentivar al alumno a que desarrolle una vida buena, alegre y feliz.

Respecto de los objetivos terminales (competencias), al término del taller los alumnos(as) estarían en condiciones de:

a) Evaluar sus propias fortalezas y debilidades en el orden de la autoestima, templanza, honestidad, empatía (respeto), prudencia y reconocimiento de la dignidad humana, según su nivel.

b) Manejar herramientas que permitan generar una estrategia personal de superación de las debilidades que atentan contra las virtudes señaladas, de la misma forma que potenciar sus fortalezas en el mismo ámbito.

c) Irradiar un mensaje positivo a su familia y al resto de sus compañeros de curso.

El taller se realizó en seis sesiones de dos horas pedagógicas cada una. En la primera etapa, se trabajó con alumnos de $1^{\circ}$ y $2^{\circ}$ medio las virtudes de la fortaleza (autoestima), templanza (ser uno mismo) y honestidad (sinceridad consigo mismo y con los demás). Con los de $3^{\circ}$ y $4^{\circ}$ medio, el respeto (empatía), la dignidad (ser persona) y la prudencia (inteligencia práctica orientada a la toma de decisiones).

El diseño instruccional del taller contempló el desarrollo de una serie de componentes, entre los que destacan un Plan de Desarrollo Secuencial por sesión y/o Módulo de Transferencia, las Guías y Protocolos de Actividades y Experiencias Prácticas, y las Estrategias Evaluativas. La efectividad tuvo que ver con el diseño y la organización de las actividades de transferencia, básicamente fundado en los siguientes criterios de aprendizaje secuencial: reconocimiento del problema (exceso o defecto), análisis de casos (análisis causa-efecto-contexto) y juicio profesional (establecimiento de principios, medios y acciones). Los recursos y metodologías utilizados fueron escogidos en razón de su funcionalidad. 


\subsection{Conferencias sobre temas de relevancia ético-social para alumnos y docentes}

El equipo de relatores se constituyó con profesores del PFG, lo que potenció la transferencia gracias a la pericia en materia de lenguaje (como viéramos antes). Además, ello permitió establecer vínculos entre docentes que, si bien trabajan en pro de un mismo objetivo, se desempeñan en instituciones de naturaleza diferente.

La metodología de las conferencias, en su primera etapa de implementación en 2004, consistió en un ciclo destinado a alumnos de $3^{\circ}$ y $4^{\circ}$ medio, en seis sesiones de trabajo de 80 minutos cada una, en las que cada expositor ocupaba alrededor de 60 minutos en la exposición dinámica del tema, siguiendo un método inductivo y desde el equilibro al exceso y el defecto, y en los 20 minutos restantes se desarrollaban dinámicas de interacción con los alumnos.

Los temas escogidos fueron especialmente atingentes a temas de familia, comienzo y término de la vida humana, y uso de la libertad y la responsabilidad. Sin embargo, resultaba de suma importancia una aproximación positiva a los mismos, de modo que el alumno, dada su situación personal, no se sintiese "agredido" por los temas. El objetivo final volvía a redundar en potenciar el ejercicio de su propia libertad y en el reconocimiento activo de la dignidad de las personas, independientemente de las circunstancias o contextos.

Para el año 2005 se estimó pertinente incorporar a todos los cursos del liceo y ofrecer ciclos de charlas por niveles $\left(1^{\circ}\right.$ a $4^{\circ}$ medio), con temas atingentes a las peculiaridades de cada edad.

\subsection{Trabajo con docentes y escuela para padres}

Los temas desarrollados con los alumnos también se trabajaron con el cuerpo de profesores del liceo y con los padres y apoderados, de modo de cerrar un círculo con todos los actores del proceso educacional. El objetivo era sintonizar a toda la comunidad del 
liceo en torno a los temas, aunque se tuvo presente la función social que cumple esta institución educacional. De este modo, el trabajo se dirigió preferentemente a temas de familia y comunicación interpersonal, con dinámicas concretas que permitiesen a padres, apoderados y profesores extraer consecuencias prácticas para aplicar en sus propias vidas.

Se dio énfasis al tema del emprendimiento, especialmente por la necesidad — detectada y evaluada — de motivar a los jóvenes a luchar por proyectos no sólo de vida sino también profesionales, desarrollar e incentivar en ellos la aspiración por ser mejores y lograr que concreten sus ideales.

\subsection{La ética como competencia laboral}

Finalmente, convencidos de que todo proyecto educativo se materializa en el aula y considerando el papel radical que tiene el docente, desde 2003 se inició un trabajo de capacitación en este tema. La primera instancia fue un taller de 22 horas, con la asistencia de todo el cuerpo de profesores del liceo.

Principalmente, se trabajó en conocer y comprender los aspectos éticos aplicados al mundo del trabajo, el concepto de ética como competencia laboral y la capacidad de manejar herramientas para desarrollar en los alumnos una conciencia ética y una cultura profesional que considere el trabajo un medio de perfeccionamiento, plenitud y felicidad.

Se trabajó en torno a la antropología del trabajo, el trabajo virtuoso en la empresa y la ética como competencia laboral, para concluir con sesiones de preparación y desarrollo de herramientas de transferencia (que alimentaron el diseño instruccional de los talleres antes expuestos): formación de personas; juicio, análisis y sentido; y la enseñanza de la ética por el método de casos.

El fundamento del trabajo es la consideración de que la auténtica finalidad de la educación es la formación integral del ser 
humano. Esta finalidad viene determinada por el sujeto sobre el que actúa, la persona, que es un todo compuesto de cuerpo, razón y espíritu. Y, siendo la persona una sola, la educación debe tender a educar a la persona toda y no sólo a alguna de sus partes (sin perjuicio de los acentos obvios que deban ponerse según de la etapa de que se trate, el proceso que se siga o el objetivo de formación profesional al que se oriente).

La ética es parte de la formación integral de la persona. Pero, dada su naturaleza, no puede ni debe agotarse en su enseñanza formal. Es necesario que los currículos establezcan instancias oficiales de aprendizaje, pero su inserción es un componente básico del proceso educativo en su conjunto.

Pero también por razones prácticas. Las virtudes no se heredan ni tampoco afloran en la persona apenas ésta asume un trabajo. El carácter se desarrolla a través de la actuación ética hasta que se transforma en un hábito, es decir, en una disposición natural al bien, que no es otra cosa que la virtud. Hay tres factores que facilitan este proceso: la práctica, el papel de los modelos y la reflexión. Todos ellos son importantes a la hora de "enseñar" ética, más allá de lo que se formule "en abstracto y lógicamente" en un curso formal.

Se trata de la teoría puesta al servicio de la acción. El peligro de poseer conocimientos que no se sabe cómo y cuándo aplicar siempre existe; pero es más riesgoso cuando dichos conocimientos atañen a la felicidad de las personas. El ser es inseparable del deber ser. Para pasar del conocimiento a la acción hay que estar convencido.

\section{Consideraciones finales}

El desafío instruccional de hacer armonizar la aplicación y el aprendizaje práctico con las nociones o contenidos mínimos que el alumno (o el profesor y/o apoderado) debía recibir, fue quizás 
lo más complejo. Hay un necesario equilibrio que debe darse entre la educación formal de la ética y una educación "informal" a través del ejemplo virtuoso y la práctica misma, que, aunque es una consecuencia del aprendizaje, también constituye un aprendizaje en sí mismo. Objetivos precisos, claridad en los niveles de competencia esperados y flexibilidad, según el contexto de que se trate, resultan claves. Importa también la coherencia entre el currículo declarado y el currículo no sólo enseñado sino apoyado, examinado y efectivamente aprendido.

Está demostrado que las personas atribuyen una importancia decisiva a los contenidos éticos de su formación. Y es que la enseñanza no puede ser neutral. Entendida como un proceso de modificación, siempre transforma para y hacia algo o, al menos, en una determinada manera. Por eso hay procesos estrictamente formales de educación: para garantizar que ciertas acciones sean realizadas por ciertas personas de cierta manera y con cierto fin, es decir, para garantizar que reaccionen de cierto modo. Ese modo es el dominio de la libertad. Toda educación, del tipo y contenido que sea, es también preparación para la vida. La ética es un elemento orientador de radical importancia en el mundo cambiante en que vivimos. Formarse, a fin de cuentas, es ganar en discernimiento espiritual.

Hablamos de ética como competencia laboral para referirnos a un saber hacer que, además, es un factor de productividad y, por ende, de competitividad. En el plano de la motivación de las acciones y la rectitud de intención, que es por definición individual, las razones para comportarse de un modo éticamente adecuado o, mejor aún, elogiable, no pueden fundarse jamás en el interés por ser más competitivo. Y ello por una causa elemental: la ética no se deja instrumentalizar. La bondad moral de la persona es un valor mucho más importante que ser competitivo en el mercado. Por lo mismo, y desde el punto de vista de los fines e intereses corporativos de una compañía, sí puede hablarse de ética como ventaja competitiva, en cuanto referencia al modo en que adecuadas disposiciones éticas 
de sus miembros y colaboradores benefician a la empresa como conjunto en sus intereses y fines específicos.

Cuando hablamos de la ética como un "valor agregado" del activo de una empresa, o "ventaja competitiva", ello debe hacerse con dos restricciones: 1) que los principios éticos que se consideran, en casos normales, como un valor agregado, deben mantenerse también cuando, en el corto plazo, su observancia puede significar algún tipo de "pérdida" material y 2) que los principios éticos que la empresa practica en sus relaciones con el "cliente externo" (clientes propiamente tales, proveedores, mercado en general, instituciones, Estado, entre otros) deben aplicarse también hacia el "cliente interno" y, en general, dentro de la propia empresa. No otra es la verdadera "actuación social de la empresa".

Estos dos criterios están muy lejos de poder, por sí solos, proveer una prueba infalible de rectitud de intención (pues no existe tal prueba). Pero sí aportan, cuando menos, un medio para prevenir el abuso del discurso ético que, pese a lo expuesto, hoy se advierte por todas partes.

A través de los programas enunciados se puede poner de manifiesto la posibilidad de llevar a cabo relaciones formativas exitosas entre instituciones de educación superior y planteles de enseñanza media técnico-profesional, pese a sus naturales diferencias. Sin duda, esto se ha visto favorecido por la sintonía en torno a una misma misión y proyecto educativo: el hecho de que, junto a la calificación técnicamente de excelencia y con alto grado de competencia, el énfasis debe estar en la formación de virtudes y hábitos. Por lo demás, en el mundo actual, estas son las competencias más apreciadas por la empresa. 